\title{
The Return of Evidence-Based Neurology to the Journal: It's All About Patient Care
}

\author{
ME Jenkins, JG Burneo
}

Can. J. Neurol. Sci. 2008; 35: 273-275

Finding the current best evidence for care of the neurological patient and keeping up-to-date with new advances in clinical research is challenging. New medical knowledge is evolving quickly, but most studies lead nowhere or are too preliminary to act on and serve to bury or at least camouflage the few important studies. Evidence Based Clinical Practice (EBCP) is becoming an important and vital tool in this rapidly changing culture. As the quantity of neurological research publications continues to expand exponentially, evidence based principles provide a systematic and comprehensive strategy to approach this literature. There is a need for more availability of evidence based critically appraised information in the clinical neurosciences.

This article will initiate a new regular section in the Canadian Journal of Neurological Sciences (now known as "The Journal”), following on from early initiatives in Evidence-Based Clinical Practice highlighted by Wiebe and colleagues in previous issues of the Journal..$^{1-3}$ This section will be dedicated to the publication of Critically Appraised Topic Summaries (CATs) in diagnosis, treatment, prognosis and other relevant aspects of the new information that is becoming available in all the subspecialties of the clinical neurosciences.

\section{What is Evidence Based Clinical Practice?}

"Evidence-based medicine is the conscientious, explicit and judicious use of the current best evidence in making decisions about the care of individual patients." ${ }^{4}$ Evidence Based Clinical Practice (EBCP) requires integration of the best available research evidence and the physician's best clinical expertise together with the patient's preferences, concerns, and expectations..$^{5}$ Although the majority of information concerning $\mathrm{EBCP}$ is focused on the evidence, in fact the expertise of the physician and the patient wishes are of equal importance in the clinical practice and implementation of EBCP. This is not a tool to be used in isolation, but a valuable skill to assist physicians in providing the optimal patient care.

The term evidence based medicine was first coined by Dr. Gordon Guyatt in 1992; ${ }^{6}$ however, the concept of EBCP was not entirely new. In the late 1970's Dr. David Sackett had started to develop the model of "critical appraisal" of the medical literature. Based on this model, a series of articles were published in CMAJ regarding the approach to critical appraisal of different types of research publications. ${ }^{7-11}$ These articles have served as the basis for the practice of EBCP. The concept was reinforced later in the JAMA giving EBCP further prominence. ${ }^{12}$ Since that time, evidence based clinical practice has become an integral part of medical school, residency and post-graduate education programs.

\section{How IS EBCP Practiced?}

There are five steps in the implementation of Evidence Based Clinical Practice: 1) developing an answerable question, 2) searching the literature, 3) critically appraising the article, 4) integrating the evidence into clinical practice, and 5) evaluating the process. ${ }^{5}$

Step One is the development of the answerable question based on a real clinical scenario. The answerable question must have a defined patient, an intervention (eg. a new diagnostic test or a medication treatment), a comparator (eg. an established treatment or a placebo), and an outcome (eg. reduction in strokes). For example, "In newly diagnosed epilepsy, how effective and how well tolerated is levetiracetam in the control of seizures compared to carbamazepine or valproic acid."

Step Two involves searching for the best available literature. This step is often the most intricate and challenging. Starting first with key words (eg. Levetiracetam AND therapy AND epilepsy) a search is undertaken in search engines such as Medline (www.ncbi.nlm.nih.gov/sites/entrez?db=pubmed), SUMsearch (http://sumsearch.uthscsa.edu), or Embase (http:// www.embase.com). In all of the search engines, limits can be applied to restrict the search to a particular type of study such as a meta-analysis (the highest level of evidence) or a randomized controlled trial (the second best level of evidence for a treatment trial). The search will usually reveal a number of articles which are then manually screened for relevance, applicability, and overall design. The best one or two articles are then chosen for appraisal.

For some topics there are methodologically sound critically appraised summaries in evidence based journals such as Bandolier (http://www.ebandolier.com) or Current Evidence (http//:www.clinicalevidence.bmj.com). In addition, the

\footnotetext{
From the Department of Clinical Neurological Sciences, University of Western Ontario, London, Ontario, Canada.

Received November 28, 2007. Final Revisions Submitted February 16, 2008. Reprint requests to: Mary Jenkins \& Jorge Burneo, Evidence-Based Neurology Programme, Department of Clinical Neurological Sciences, University of Western Ontario, 339 Windermere Rd, London, ON, N6A5A5, Canada.
} 
Cochrane Library (http://www.theCochraneLibrary.com) presents evidence based systematic reviews on some topics. However in most cases these completed summaries do not exist, especially for neurological disorders.

Step Three entails the critical appraisal of the chosen articles. The article is assessed following the CMAJ and JAMA guidelines developed by Guyatt and Sackett. ${ }^{12}$ These guidelines provide systematic tools to assess the validity of the study design (was the study well enough executed to answer the question?), the magnitude of the results (are the results clinically important?), and the applicability to the initial clinical case (does this apply to your clinical practice?).

Step Four is the integration of the critically appraised evidence with the physician's clinical expertise and the patient's wishes. This is the most important step in the utilization of evidence based clinical practice and involves consideration of the patient's expectations, concerns and preferences in the context of the best evidence.

Step Five is a self-monitoring procedure that encompasses the evaluation of the entire process and the development of strategies to improve evidence based skills in the future. ${ }^{5}$

\section{Why is Evidence Based Clinical Practice Important?}

The clinical practice of evidence based medicine in neurology has been slow to develop but many more publications directed at evidence based neurology are appearing. In addition, through medical school and residency programs, evidence based clinical practice is being taught and utilized in day to day practice. ${ }^{13}$ There are limitations to evidence based practice and many are inherent to the practice of medicine. Particularly in neurology, where many diseases are rare, there is a paucity of well-designed trials. As with any clinical tool, it is often difficult to apply the results to an individual patient. ${ }^{5}$ Time-constraints are a frequent deterrent to the utilization of evidence based principles in clinical practice ${ }^{14}$ Lastly, there is a lack of evidence to support that using EBCP will actually improve patient care. There is however, a growing research to examine this issue. A recent study looked at the effects of employing EBCP principles in a medicine inpatient service on the length of hospital stay. The study found that including evidence based practice strategies in daily morning rounds decreased the mean length of stay from five days to three days. ${ }^{15}$ In our current health care crisis with lack of resources, this type of strategy could have huge implications, not just for patient care on an individual basis but also for the entire health care system.

\section{Why the Clinical Neurosciences?}

Neurologists and neurosurgeons are constantly confronted with clinical problems. Historically, solutions have been found in textbooks, or the oral and written traditions of the hospital. In addition, junior physicians, residents and students would trust their most senior colleagues because of their ability to understand neurophysiology and to apply this knowledge to the problem at hand. This paradigm of education and practice resulted in wide variation in the approach to clinical problems across institutions (or even across different authorities and their services within an institution), and patients would experience different outcomes, not all of them optimal.
At the same time, clinician-neuroscientists conducted and published important clinical research which would potentially help decide on the best management strategies leading to the best patient outcomes. However, neither practitioners nor the authorities on whom they relied were equipped with the attitudes and skills to assess the value of these research findings or to judiciously incorporate this evidence into clinical practice. Thus, clinical neuroscience research found suboptimal use. ${ }^{16}$

Within other areas of medicine evidence based information services have been developed that provide a means for organizing and accessing current best evidence in a timely manner. These types of comprehensive evidence based information, unfortunately, have not been as readily available for the clinical neurosciences. ${ }^{17}$ Thus, knowledge of EBCP is highly relevant to clinicians in the neurosciences and there is a need for collections of good quality, peer-reviewed, EBCP neurological statements, such as Critically Appraised Topic Summaries.

At the University of Western Ontario, evidence based neurology has become an integral part of the residency program. The evidence based neurology program was initially conceived by Dr. Samuel Wiebe in 1999, and is now under the direction of Dr. Mary Jenkins and Dr. Jorge Burneo. This program meets on a regular basis to critically appraise the evidence regarding neurological disorders. At the completion of each session, a Critically Appraised Topic Summary is developed and posted on the web site. . $^{1,18}$

\section{A New Section at the Journal: The CaTs Have Arrived}

This new section of the Journal will present peer-reviewed CATs dealing with current research in clinical neurosciences. Each CAT will appraise one or two recent research articles dealing with a particular topic. All readers, particularly evidencebased neurosciences programs are invited to submit their CATs. In addition, meta-analyses and systematic reviews will also be considered for publication if they are pertaining to evidencebased neurological/neurosurgical practice, and are considered appropriate by the Co-Editors of this section and the Editor-InChief of the Journal.

The CAT will need to be prepared systematically, following guidelines and will provide objective answers derived from a thorough, rigorous process, as previously published in this journal and a number of others., ${ }^{1,418,19}$ They will be subject to standard peer-reviewed process as any article published in this journal. The CAT is a concise summary of the critical appraisal process of reviewing an article, following the steps one through five outlined above. Usually a CAT will arise from a journal club encompassing the process of review and conclusions reached from the group discussion. The CAT has a very structured format to allow for quick access to the "best-evidence".

A complete CAT is a one or two page summary that includes all of the following:

1. A brief title that summarizes the conclusion reached about the article.

2. Clinical Bottom Lines consisting of short statements summarizing the key "take-home" points.

3. The clinical problem which cues the reader to the nature of the case. The clinical problem comes from real life dilemmas that are faced by clinicians. 
4. The clinical question includes the patient, intervention, comparator, and outcome.

5. The search strategy - including search terms, search engines used, and the reasons why the article chosen is the best evidence for the clinical question.

6. The evidence is described briefly including the type of study, patient population, and outcomes reported for the article reviewed.

7. The data is usually presented in tabular form and highlights the clinically significant data such as number needed to treat, specificity, hazard ratios etc.

8. Comments are added regarding the quality of the study and any concerns which were identified by the critical appraisal process.

9. The reference, the appraiser, the date appraised, and the date expired.

10. Lastly, it will include a clinical comment from an "expert" on the particular topic.

The CATs have many advantages. They are a concise, complete, rigorous, and systematic review of a research article. They are designed to provide enough information to quickly determine the bottom lines and apply this information to the appropriate clinical setting. They are a great time saver. Critically Appraised Topic Summaries are not without disadvantages. They only present the appraisal of one or two articles of the "best evidence" and do not systematically review all articles on a topic. In addition, they are only as current as the day they were made and even the best CATs eventually expire. Dates to review the CAT will be included to hopefully circumvent this problem. ${ }^{19}$

The CATs published in the Journal will be peer-reviewed prior to publication. The majority of CATs posted on web-sites do not undergo a formal peer-review process but we feel that this will enhance the validity of the CATs. Recommendations for the formal review of CATs have been developed by other authors..$^{20,21}$ We will encourage reviewers to use these references in addition to their own expertise in Evidence Based Clinical Practice to guide the peer-review process. The expectation is that a complete CAT will contain all of the items listed above. Further, reviewers will assess the quality and relevance of the clinical question, search strategy, article choice, appraisal of the study design, discussion of all importance outcomes, and analysis of data. Finally, the clinical implications and correctness of the bottom lines and title will be reviewed..$^{20,21}$

We look forward to this exciting new section in the Journal and we hope this will allow more availability to the best evidence in clinical neurosciences.

\section{REFERENCES}

1. Wiebe S. An evidence based approach to the first unprovoked seizure. Can J Neurol Sci. 2002;29(2):120-4.

2. Bussiere $\mathrm{M}$, Wiebe $\mathrm{S}$. Progress in clinical neurosciences: measuring the benefits of therapies for neurological disorders. Can J Neurol Sci. 2005;32(4):419-24.

3. Bussiere $\mathrm{M}$, Wiebe $\mathrm{S}$. The numbers needed to treat for neurological disorders. Can J Neurol Sci. 2005;32(4):440-9.

4. Sackett DL, Rosenberg WM, Gray JA, Haynes RB, Richardson WS. Evidence based medicine: what it is and what it isn't. BMJ. 1996;312(7023):71-2.

5. Sackett DL, Strauss SE, Richardson WS, Rosenberg W, Haynes RB. Evidence based medicine: how to practice and teach EBM. 3rd ed. London: Churchill Livingstone; 2003.

6. Evidence-based medicine. A new approach to teaching the practice of medicine. Evidence-Based Medicine Working Group. JAMA. 1992;268(17):2420-5.

7. How to read clinical journals: IV. To determine etiology or causation. CMAJ. 1981;124(8):985-90.

8. How to read clinical journals: V. To distinguish useful from useless or even harmful therapy. CMAJ. 1981;124(9):1156-62.

9. How to read clinical journals: III. To learn the clinical course and prognosis of disease. CMAJ. 1981;124(7):869-72.

10. How to read clinical journals: I. Why to read them and how to start reading them critically. CMAJ. 1981;124(5):555-8.

11. How to read clinical journals: II. To learn about a diagnostic test. CMAJ. 1981;124(6):703-10.

12. Guyatt GH, Rennie D. User's guide to the medical literature. Chicago: AMA Press; 2002.

13. Amin L, Burneo JG, Jenkins ME. Evaluation of a novel educational strategy for integrating evidence based neurology into clinical practice [Abstract]. Neurology. 2007;68 Suppl 1:A76.

14. Burneo JG, Jenkins ME, Bussiere M. Evaluating a formal evidencebased clinical practice curriculum in a neurology residency program. J Neurol Sci. 2006;250(1-2):10-9.

15. Banks DE, Shi R, Timm DF, Christopher KA, Duggar DC, Comegys M, et al. Decreased hospital length of stay associated with presentation of cases at morning report with librarian support. J Med Libr Assoc. 2007;95(4):381-7.

16. Montori VM, Guyatt GH. What is evidence-based medicine? Endocrinol Metab Clin North Am. 2002;31(3):521-6, vii.

17. Wiebe S, Demaerschalk B. Evidence based care in the neurosciences. Can J Neurol Sci. 2002;29(2):115-9.

18. http://www.uwo.ca/cns/ebn/. (Accessed at November 20, 2007.

19. Wingerchuk DM, Demaerschalk BM. Critically appraised topics: the evidence-based neurologist. Neurologist. 2007;13(1):1.

20. Dawes M. Critically appraised topics and evidence-based medicine journals. Singapore Med J. 2005;46(9):442-8; quiz 9.

21. Coomarasamy A, Latthe P, Papaioannou S, Publicover M, Gee H, Khan KS. Critical appraisal in clinical practice: sometimes irrelevant, occasionally invalid. J R Soc Med. 2001;94(11): 573-7. 